Research Paper

\title{
FEZF1 is an Independent Predictive Factor for Recurrence and Promotes Cell Proliferation and Migration in Cervical Cancer
}

\author{
Yang Lan, Xuewei Xiao, Yu Luo, Zhengchi He, Xu Song ${ }^{凶}$ \\ Key Laboratory of Bio-Resource and Eco-Environment of Ministry of Education, College of Life Sciences, Sichuan University, Chengdu 610065, Sichuan, \\ P.R.China \\ $\triangle$ Corresponding author: Xu Song, College of Life Sciences, Sichuan University, No. 29 Wangjiang Road, Wuhou District, Chengdu 610065, Sichuan, China. Tel: \\ +86-28-8541 0032; Fax: +86-28-8541 8926; E-mail: xusong@scu.edu.cn \\ (C) Ivyspring International Publisher. This is an open access article distributed under the terms of the Creative Commons Attribution (CC BY-NC) license \\ (https://creativecommons.org/licenses/by-nc/4.0/). See http://ivyspring.com/terms for full terms and conditions.
}

Received: 2018.03.15; Accepted: 2018.08.06; Published: 2018.10.10

\begin{abstract}
The Fez family zinc finger protein 1 (FEZFI), a critical transcription factor in nervous system development, has been implicated in cancer progression recently. However, its clinical significance remains unknown. By analyzing gene expression data of eight most common cancer types from The Cancer Genome Atlas (TCGA), we found that FEZFI prominently associated with the recurrence-free survival of cervical cancer patients $(P<0.001)$ and was an independent diagnostic factor for cervical cancer recurrence $(P=0.002)$. Moreover, FEZFI expression was significantly higher in the tumor samples from cervical cancer patients with relapse in TCGA $(P=0.015)$. By RNA interference, we knocked down FEZFI and found that cell proliferation, growth and migration were significantly decreased in C33A and $\mathrm{SiHa}$ cells. Meanwhile, FEZF1 knockdown also attenuated the growth of C33A cells in nude mice. In contrast, expression of FEZF1 promoted cell proliferation, growth and migration in HeLa cells. Using chromatin immunoprecipitation (ChIP) assay, we revealed that FEZFI could bind to multiple key genes in the Wnt signaling pathway in HeLa cells. Furthermore, analysis of the levels of $\beta$-catenin protein, the core component of the Wnt pathway, and downstream effector genes of the pathway showed that FEZFI could activate the Wnt pathway. Together, these results suggest that FEZFI promotes cell proliferation and migration possibly by acting as a transcriptional activator of the Wnt signaling pathway in cervical cancer, and also provide a valuable molecular predictive marker for cervical cancer recurrence.
\end{abstract}

Key words: FEZF1; Cervical Cancer; Recurrence; Diagnostic Factor; Wnt Signaling Pathway

\section{Introduction}

Cervical cancer is the fourth most frequently diagnosed cancer and the fourth leading cause of cancer death in women worldwide, with an estimated 527,600 new cases and 265,700 deaths occurred in 2012 [1]. Of these, less economically developed countries account for $\sim 84 \%$ of cases and $\sim 87 \%$ of deaths [1]. In more developed countries, the widespread use of Papanicolaou smear screening, which allows early detection and treatment of pre-cancerous lesion, has dramatically declined the incidence rate [2]; however, it is still the ninth leading cause of cancer death in the developed regions and an estimated 35,500 women's lives were still threatened by this disease in 2012 [1]. The prognosis of cervical cancer largely depends on the stage at diagnosis [3]. Patients with early-stage disease (FIGO stage I and II) have a high cure rate of $80-95 \%$, but the rate drops to $60 \%$ for stage III patients [3]. Recurrence is another poor prognosis factor for cervical cancer [4]. The recurrence rate ranges from $8 \%$ to $26 \%$, and most of recurrences $(62-89 \%)$ occur within the first two years after primary treatment [5]. The 5-year overall survival rate after recurrence (SAR) is only $10.1 \%$ to $22.3 \%$ [6, 7]. Early detection of recurrence has been supposed to improve the survival 
of the patients [5]. However, the benefit of salvage therapy for recurrence is still limited and the availability of treatment options largely depends on previous therapy received [8]. Therefore, identification of prognostic factors, which could predict recurrence and allow appropriate primary treatment for patients with high-risk, is of great importance for improving the outcome of cervical cancer patients.

The Fez family zinc finger protein 1 (FEZF1) is a $\mathrm{C} 2 \mathrm{H} 2$ zinc finger transcription factor that plays critical roles during forebrain and olfactory system development in vertebrates [9]. In mice, Fezf1 binds to and represses the expression of Hes5, a transcription factor that inhibits neuronal differentiation, to facilitate neurogenesis in the forebrain [10]. Recently, FEZF1 has also been implicated in the progression of human cancers [11, 12]. A 2- to 20-fold increase of FEZF1 expression was observed in more than $50 \%$ of gastric cancer tissues when compared to their adjacent normal tissues [11]. Then, the upregulation of FEZF1 was also reported in colorectal cancer [12]. In gastric cancer cells, FEZF1 was found to enhance proliferation and tumorigenic ability by binding to and activating oncogene K-ras [11]. In addition, FEZF1 was also shown to promote cell migration and invasiveness in colorectal cancer cells [12]. However, the clinical significance of FEZF1 and its target genes in human cancers are still largely unknown.

In present study, we revealed the prominently clinical significance of FEZF1 in cervical cancer by analyzing gene expression data of eight most common cancer types from The Cancer Genome Atlas (TCGA). The effects of FEZF1 on cervical cancer cells were examined both in vitro and in vivo. Moreover, we also demonstrated that FEZF1 may exert its function by binding to and activating genes in the Wnt signaling pathway.

\section{Materials and Methods}

\section{TCGA datasets}

Clinical and RNAseq gene expression (HiSeqV2) data of eight cancer types, including breast cancer (BRCA), cervical cancer (CESC), colon and rectal cancer (COADREAD), esophageal cancer (ESCA), liver cancer (LIHC), lung cancer (LUNG), prostate cancer (PRAD) and stomach cancer (STAD) were downloaded from the TCGA hub, UCSC Xena (https://xenabrowser.net/datapages/). Only data of the primary tumor samples were used for analysis in this study. In survival analysis for CESC, COADREAD, ESCA, LUNG and STAD, patients with detectable FEZF1 expression, overall survival (OS) and recurrence-free survival (RFS) data were included. The patients were divided into high- and low-FEZF1 expression groups according to the Youden index determined using FEZF1 expression values and OS data. For BRCA, LIHC and PRAD, patients with both OS and RFS data were included. And the patients were simply divided in to FEZF1-present or -absent groups. To analysis the associations between FEZF1 expression and the clinicopathological characteristics in CESC, five patients with undefined clinical stage were excluded, and Cox proportional hazards regression analysis was carried out with the same set of CESC patients.

\section{Cell culture}

Human cervical cancer cell lines C33A, SiHa and HeLa, and embryonic kidney cell line 293T (HEK 293T) were purchased from the Cell bank of the Chinese Academy of Sciences (Shanghai, China). C33A cells were cultured in MEM (Gibco, Grand Island, NY, USA). SiHa, HeLa and HEK293T cells were cultured in DMEM (Gibco). All media were supplemented with $10 \%$ fetal bovine serum (FBS, ExCell, Hangzhou, China), $100 \mathrm{U} / \mathrm{ml}$ penicillin and $100 \mu \mathrm{g} / \mathrm{ml}$ streptomycin (HyClone, Logan, UT, USA).

Cells were transfected with Lipofectamine 2000 (Invitrogen, Carlsbad, CA, USA) following the manufacturer's protocol. For generation of short hairpin RNA (shRNA) lentiviruses, HEK293T cells were co-transfected with $\mathrm{pLKO} .1$ shRNA vectors and packaging plasmids, psPAX2 and pMD2.G. For FEZF1 knockdown, C33A and SiHa cells were transduced with shRNA lentiviruses and selected by 1 $\mu \mathrm{g} / \mathrm{ml}$ puromycin (Sigma-Aldrich, St Louis, MO, USA) for 3 days. After selection, cell pools were grown in culture media without puromycin for $24 \mathrm{~h}$ before further analysis. For the expression of FEZF1, HeLa cells were transfected with FEZF1 expression plasmid and subjected to analysis $24 \mathrm{~h}$ after transfection.

\section{Plasmid construction}

For RNA interference, shRNA expression vectors were generated by annealing and inserting shRNA oligonucleotide pairs into $\mathrm{pLKO} .1$ vector. The target sequences of shFEZF1-1 and shFEZF1-2 are 5'GGTC TTTAATGCGCACTAT3' and 5'GTGGTTCATTATG GATGAT3', respectively. The sequence in the nontarget control shCTRL is $5^{\prime}$ CAACAGCCACAACGTC TAT3'. For the expression of FEZF1, the human codon-optimized cDNA sequence of FEZF1 open reading frame (ORF) was synthesized by Genewiz Inc. (Suzhou, China) with a FLAG tag sequence fused in-frame to the 3' end of the ORF. Then, the cDNA was cloned into $\mathrm{pEGFP-C1}$ and fused in-frame to the C-terminal of the EGFP ORF (pEGFP-FEZF1). 


\section{Reverse transcription-quantitative PCR (RT-qPCR)}

RNA was extracted using TRIzol reagent (Invitrogen), treated with DNase I (Thermo Fisher Scientific, Rockford, IL, USA) and converted into cDNA using random hexamer primers and RevertAid M-MuLV reverse transcriptase (Thermo Fisher Scientific). cDNA was quantified using SYBR green I master mix (Foregene, Chengdu, China) and genespecific primers on the StepOnePlus System (Applied Biosystems, Foster City, CA, USA). PCR amplification reactions were all run in triplicates for each cDNA sample. For comparison, RNA level was first normalized to B2M mRNA, and the relative level of RNA was determined by setting controls as 1 . The qPCR primers are listed in Table S1.

\section{Western blot analysis}

Western blot was performed according to standard methods. In brief, proteins were separated by SDS polyacrylamide gel electrophoresis (SDSPAGE), transferred onto PVDF membranes (Bio-Rad Laboratories, Hercules, CA, USA) and incubated overnight at $4{ }^{\circ} \mathrm{C}$ with corresponding antibodies: antiFLAG M2-HRP (1:2000; A8592, Sigma-Aldrich), anti-GAPDH-HRP (1:30000; HRP-60004, Proteintech, Chicago, IL, USA) and anti- $\beta$-catenin (1:1000; 617832, Zen BioScience, Chengdu, China). An HRPconjugated sheep anti-rabbit IgG secondary antibody (1:5000; SA00001-2, Proteintech) was also used for the detection of $\beta$-catenin. Protein signal was detected using ECL chemiluminiscent substrate (Thermo Fisher Scientific).

\section{Cell Counting Kit-8 (CCK-8) and colony formation assay}

Cell proliferation was measured by CCK-8 (Dojindo Laboratories, Kumamoto, Japan) according to the manufacturer's recommendations. Briefly, C33A, SiHa and HeLa cells were seeded in triplicates in 96-well plates at 7000, 2000 and 2000 cells/well, respectively. Cell culture media were changed into fresh media containing $10 \%(\mathrm{v} / \mathrm{v})$ CCK-8 reagent at indicated times. After a $2 \mathrm{~h}$ incubation under the culture condition, the absorbance at $450 \mathrm{~nm}$ of each well was measured on a microplate reader. The mean value of the wells with media alone was used as background and was subtracted from the absorbances of the wells containing cells.

For colony formation assay, $\mathrm{C} 33 \mathrm{~A}, \mathrm{SiHa}$ and HeLa cells were seeded at 600, 600 and 400 cells/well respectively in 6-well plates and cultured for 12 days. Cell colonies were fixed with $4 \%$ paraformaldehyde for $10 \mathrm{~min}$, stained with $0.1 \%$ crystal violet for $5 \mathrm{~min}$ and washed twice with phosphate-buffered saline
(PBS). The number of colonies containing more than 50 cells was counted.

\section{Transwell migration assay}

For migration assay, Transwell-24 Well Permeable Supports (Corning, Bedford, MA, USA) with $8 \mu \mathrm{m}$ pores were used. C33A $\left(1 \times 10^{5}\right.$ cells $)$, SiHa $\left(2 \times 10^{4}\right.$ cells $)$ and HeLa $\left(5 \times 10^{4}\right.$ cells $)$ cells were seeded in the upper chambers with media containing $1 \%$ FBS. The lower chambers were filled with media containing 20\% FBS. After cultured for $20 \mathrm{~h}$, cells on the upper side of the support membranes were wiped out with a cotton swab. Cells on the lower side were fixed with $4 \%$ paraformaldehyde for $15 \mathrm{~min}$, stained with $1 \%$ crystal violet for $10 \mathrm{~min}$ and counted under a microscope (Olympus).

\section{Tumorigenesis in nude mice}

Female BALB/c nude mice (5 weeks old) were housed and maintained under special pathogen-free (SPF) condition. C33A cells $\left(5 \times 10^{6}\right)$ were injected subcutaneously into right flanks of nude mice $(\mathrm{n}=6$ for each group). Tumors were measured every 3 days with a slide caliper and tumor volumes were calculated by the formula: volume $\left(\mathrm{mm}^{3}\right)=0.5$ $\times$ length $\times$ width $^{2}$. After 30 days, mice were sacrificed, and tumors were dissected, photographed, and weighted.

\section{Gene ontology (GO) enrichment analysis and Pathview visualization}

ChIP (chromatin immunoprecipitation)-seq data of FEZF1 under series accession number GSE76495 were obtained from Gene Expression Omnibus (GEO). To reduce the background, only binding sites with scores over the mean were included for further analysis. GO enrichment analysis of the FEZF1binding genes was performed using clusterProfiler [13], an R/Bioconductor package. Then, the redundancy of the enriched GO terms was reduced using GOSemSim package, which computes the semantic similarity among GO terms [14]. Pathview package based on KEGG pathways [15] was used for the visualization of FEZF1-binding genes in the Wnt signaling pathway.

\section{ChIP assay}

HeLa cells $\left(2 \times 10^{7}\right.$ cells $)$ were crosslinked in $1 \%$ formaldehyde for $30 \mathrm{~min}$ at room temperature. The crosslinking was quenched by glycine at a final concentration of $0.125 \mathrm{M}$ for $5 \mathrm{~min}$. The crosslinked cells were sonicated for $20 \mathrm{~min}$ on ice in $1 \mathrm{ml}$ ChIP buffer ( $25 \mathrm{mM}$ Tris [pH 8.0], $0.15 \mathrm{M} \mathrm{NaCl}, 0.5 \% \mathrm{NP}-40$, $0.1 \%$ SDS, $0.5 \mathrm{mM}$ DTT and $1 \times$ complete protease inhibitors [Roche, Indianapolis, IN, USA]). Then, cell lysates were incubated with $50 \mu \mathrm{l}$ anti-FLAG M2 
magnetic beads (Sigma-Aldrich) at $4^{\circ} \mathrm{C}$ overnight with rotation. After five washes with ChIP buffer, the beads were resuspended in $0.2 \mathrm{ml}$ SDS buffer $(25 \mathrm{mM}$ Tris [pH 8.0], $0.2 \mathrm{M} \mathrm{NaCl}$ and $1 \%$ SDS) to elute the protein-DNA complexes. Then, crosslink was reversed at $65^{\circ} \mathrm{C}$ overnight, and DNA was recovered by phenol/chloroform extraction and subjected to qPCR analysis. The primers used in qPCR are also listed in Table S1.

\section{Statistical analysis}

All statistical analyses were carried out using SPSS Statistics version 20 (IBM). Data are presented as mean \pm standard deviation of three independent experiments. Differences in patient survival were assessed by the Kaplan-Meier method and compared using the log-rank test. The differences of means between groups were determined using two-tailed Student t-test or one-way analysis of variance (ANOVA). The correlation between FEZF1 level and the clinicopathological characteristics was analyzed using two-tailed Pearson's chi-square test. Univariate and multivariate Cox proportional hazards models were utilized to estimate the relative risk ratio for each factor. $P<0.05$ was considered statistically significant. ${ }^{*} P<0.5$ and ${ }^{* *} P<0.01$.

\section{Results}

\section{FEZFI expression prominently associated with the RFS of cervical cancer patients in TCGA}

We first examined FEZF1 expression in eight types of human cancers with high incidence and mortality rates worldwide, including BRCA, CESC, COADREAD, ESCA, LIHC, LUNG, PRAD and STAD, using RNAseq gene expression data from TCGA. FEZF1 was detected in most of primary tumor samples from CESC, COADREAD, ESCA, LUNG and STAD, but only in a small portion of the samples from BRCA, LIHC and PRAD (Figure 1A). To explore its clinical relevance, we analyzed the relation of FEZF1 expression with the OS and RFS of patients in all these eight cancer types by Kaplan-Meier method. The results showed that FEZF1 expression significantly associated with the OS of CESC, ESCA and LUNG patients, as well as the RFS of CESC and PRAD patients, but no significant association was observed in other cancer types (Figure S1), including COADREAD and STAD, in which FEZF1 has been reported to enhance cancer cell aggressiveness. Among all these cancer types, the expression of FEZF1 exhibited a prominent influence on the survival of CESC patients (Figure 1B). The 5-year OS rates for patients expressing highand low-level of FEZF1 were $47 \%$ and $75.8 \%$ in CESC, respectively. Meanwhile, the 5-year RFS rate was $89.9 \%$ for patients with low level of FEZF1 and drops to only $22 \%$ for patients with high level of FEZF1. Thus, we focused on and further characterized the clinical significance of FEZF1 in cervical cancer.

\section{FEZF I was an independent diagnostic factor for cervical cancer recurrence in TCGA patients}

We first examined FEZF1 expression in CESC patients with different clinicopathological characteristics. The results demonstrated that FEZF1 expressed at a significantly higher level in the samples from patients 
with relapse compared to the samples from patients without diagnosed recurrence ( $P=0.015$; Figure $1 C)$, but no difference of FEZF1 expression was observed between patient groups with other characteristics, including age, histologic type, histologic grade, clinical stage, pathological $\mathrm{T}$ stage and pathological $\mathrm{N}$ stage (Table S2). We also examined the correlation between FEZF1 level and the clinicopathological characteristics. Similarly, high level of FEZF1 expression associated with recurrence $(P=0.003$, Chi-square test) but not with the other characteristics (Table S3). By univariate Cox proportional hazards regression analysis, we found that clinical stage, pathological T stage, pathological N stage and FEZF1 expression were prognostic factors for the OS of CESC patients, and pathological $\mathrm{N}$ stage and FEZF1 expression were prognostic factors for the RFS of CESC patients (Table 1). Then, we performed multivariate analysis for these risk factors. The results showed that FEZF1 expression was an independent diagnostic factor for both the OS and RFS of CESC patients (Table 2). When taking recurrence into account in the multivariate analysis for the OS, FEZF1 expression was no longer an independent risk factor (Table S4), which suggests that FEZF1 influences the OS through its effect on recurrence. Overall, we demonstrated that FEZF1 expression associated with recurrence and was an independent diagnostic marker for recurrence in cervical cancer. Patients with high level of FEZF1 were at higher risk of recurrence $(\mathrm{HR}=3.479 ; 95 \% \mathrm{CI}, 1.553-7.795 ; P=0.002)$ than patients with low level of FEZF1 expression (Table 2).

\section{FEZF1 knockdown inhibited cervical cancer cell proliferation, growth and migration}

To investigate the function of FEZF1 in cervical cancer cells, we knocked down FEZF1 in C33A and $\mathrm{SiHa}$ cells by RNA interference using two independent shRNAs (Figure 2A). We first measured the effect of FEZF1 on cell proliferation by CCK-8 assay. The results showed that FEZF1 knockdown significantly decreased cell proliferation in C33A and SiHa cells (Figure 2B). Then, colony formation assay was performed to measure the continued growth capacity of these cells. As shown in Figure 2C, FEZF1 knockdown cells formed significantly less colonies compared to their control cells. We also examined the effect of FEZF1 on cell migration by Transwell assay. The results showed that cell migration ability was attenuated by FEZF1 knockdown in C33A and SiHa cells (Figure 2D).
A

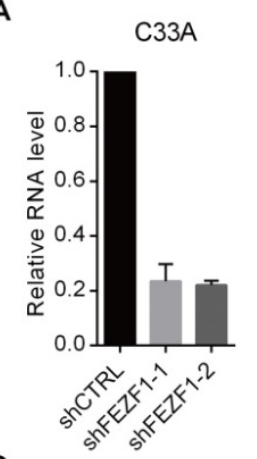

C

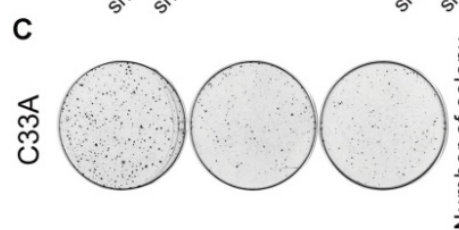

shCTRL shFEZF1-1 shFEZF1-2

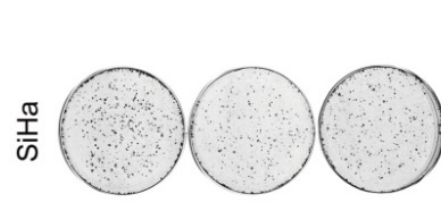

shCTRL shFEZF1-1 shFEZF1-2
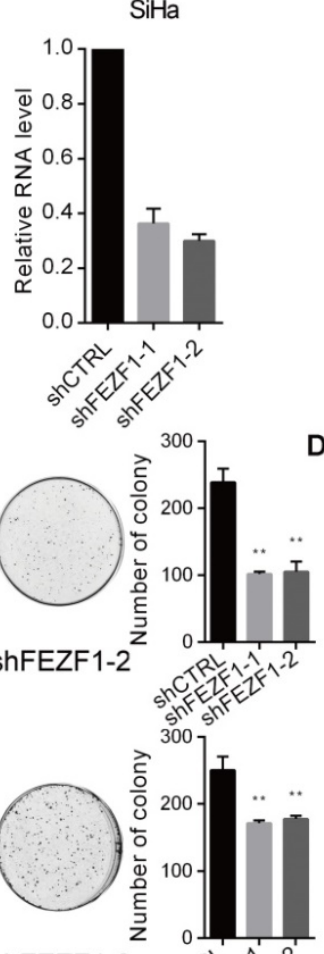

年 scis
B
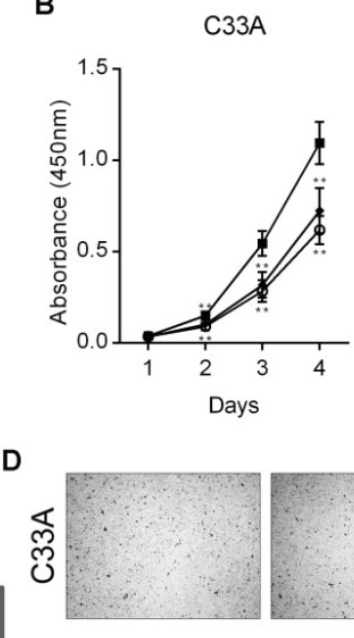

shCTRL

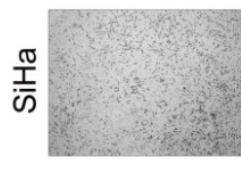

shCTRL
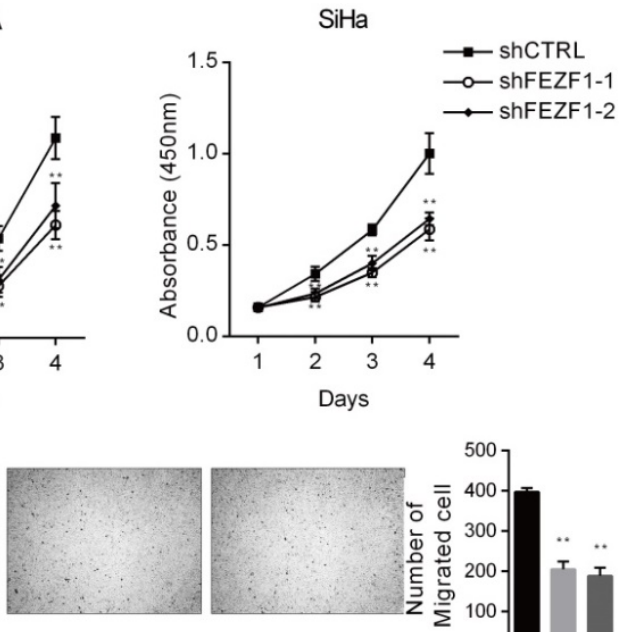

shFEZF1-1

shFEZF1-2

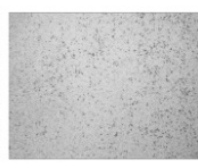

shFEZF1-1

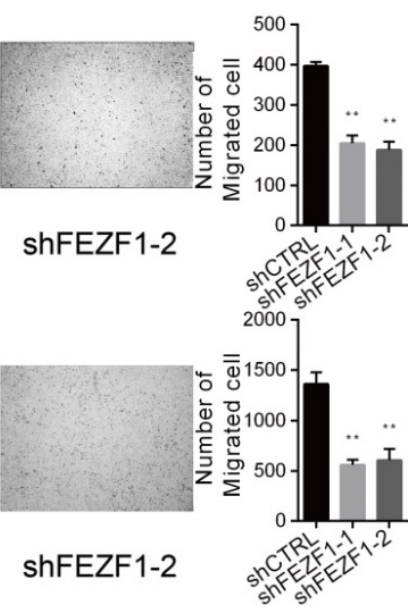

Figure 2. FEZFI knockdown inhibited cervical cancer cell proliferation, growth and migration. (A) RT-qPCR analysis showed efficient knockdown of FEZF1 by two independent shRNAs in C33A and SiHa cells. (B) Cell Counting Kit-8 assay demonstrated that cell proliferation was significantly decreased after FEZF1 knockdown in C33A and SiHa cells. (C) Colony formation assay showed that FEZF1 knockdown C33A and SiHa cells formed less colonies compared to their control cells. (D) Transwell migration assay revealed that the knockdown of FEZF1 attenuated the migration of C33A and SiHa cells. Data represent mean \pm SD of three independent experiments. $* P<0.05, * * P<0.01$. 
Table 1. Univariate Cox proportional hazards regression analysis of the OS and RFS of the CESC patients from TCGA

\begin{tabular}{|c|c|c|c|c|c|c|}
\hline \multirow[t]{2}{*}{ Characteristics } & & \multirow[t]{2}{*}{$\mathbf{N}$} & \multicolumn{2}{|l|}{ OS } & \multicolumn{2}{|l|}{ RFS } \\
\hline & & & HR $(95 \%$ CI) & $P$ & HR (95\% CI) & $P$ \\
\hline \multirow[t]{2}{*}{ Age } & $<50$ & 115 & 1 & & 1 & \\
\hline & $\geq 50$ & 79 & $1.340(0.741-2.423)$ & 0.334 & $0.993(0.450-2.191)$ & 0.986 \\
\hline \multirow[t]{2}{*}{ Histologic type } & Squamous cell & 160 & 1 & & 1 & \\
\hline & Other types & 34 & $0.845(0.356-2.005)$ & 0.703 & $2.114(0.880-5.075)$ & 0.094 \\
\hline \multirow[t]{3}{*}{ Histologic grade } & G1-2 & 103 & 1 & & 1 & \\
\hline & G3 & 68 & $0.748(0.360-1.554)$ & 0.436 & $1.620(0.723-3.630)$ & 0.241 \\
\hline & GX† & 23 & $1.748(0.789-3.872)$ & 0.169 & $0.406(0.053-3.106)$ & 0.385 \\
\hline \multirow[t]{2}{*}{ Clinical stage } & I-II & 153 & 1 & & 1 & \\
\hline & III-IV & 41 & $2.727(1.485-5.009)$ & $0.001^{\star *}$ & $1.036(0.388-2.763)$ & 0.944 \\
\hline \multirow[t]{3}{*}{ Pathological T stage } & $\mathrm{T} 1-2$ & 138 & 1 & & 1 & \\
\hline & T3-4 & 21 & 4.390(2.037-9.459) & $<0.001^{\star *}$ & $1.984(0.662-5.948)$ & 0.221 \\
\hline & $\mathrm{TX}^{\dagger}$ & 35 & $2.618(1.315-5.212)$ & $0.006^{\star *}$ & $1.172(0.425-3.236)$ & 0.759 \\
\hline \multirow[t]{3}{*}{ Pathological N stage } & No & 86 & 1 & & 1 & \\
\hline & N1 & 35 & $6.833(2.590-18.028)$ & $<0.001^{\star *}$ & 5.164(1.874-14.230) & $0.002^{* *}$ \\
\hline & $\mathrm{NX}^{\dagger}$ & 73 & $5.184(2.125-12.646)$ & $<0.001^{\star * *}$ & $1.855(0.660-5.215)$ & 0.241 \\
\hline \multirow[t]{2}{*}{ FEZF1 } & Low & 147 & 1 & & 1 & \\
\hline & High & 47 & $2.032(1.077-3.834)$ & $0.029^{\star \star *}$ & 3.604(1.613-8.051) & $0.002^{* *}$ \\
\hline
\end{tabular}

Abbreviations: $\mathrm{HR}=$ hazard ratio; $95 \% \mathrm{CI}=95 \%$ confidence interval. ${ }^{\dagger} \mathrm{GX}$, TX and NX: samples with unknown histological grade, tumor or node stage. ${ }^{*} P<0.05,{ }^{* *} P<0.01$.

Table 2. Multivariate Cox proportional hazards regression analysis of the OS and RFS of the CESC patients from TCGA

\begin{tabular}{|c|c|c|c|c|c|c|}
\hline \multirow[t]{2}{*}{ Characteristics } & & \multirow[t]{2}{*}{$\mathbf{N}$} & \multicolumn{2}{|l|}{ OS } & \multicolumn{2}{|l|}{ RFS } \\
\hline & & & HR $(95 \%$ CI) & $P$ & HR $(95 \%$ CI) & $P$ \\
\hline \multirow[t]{2}{*}{ Clinical stage } & I-II & 153 & 1 & & & \\
\hline & III-IV & 41 & $1.327(0.625-2.819)$ & 0.462 & & \\
\hline \multirow[t]{3}{*}{ Pathological T stage } & $\mathrm{T} 1-2$ & 138 & 1 & & & \\
\hline & T3-4 & 21 & $3.250(1.151-9.175)$ & $0.026^{*}$ & & \\
\hline & $\mathrm{TX}+$ & 35 & $2.302(0.811-6.532)$ & 0.117 & & \\
\hline \multirow[t]{3}{*}{ Pathological N stage } & N0 & 86 & 1 & & 1 & \\
\hline & N1 & 35 & $5.732(2.137-15.374)$ & $0.001^{* *}$ & $4.903(1.777-13.526)$ & $0.002^{* *}$ \\
\hline & $\mathrm{NX}+$ & 73 & $2.375(0.753-7.494)$ & 0.140 & $1.675(0.594-4.725)$ & 0.330 \\
\hline \multirow[t]{2}{*}{ FEZF1 } & Low & 147 & 1 & & 1 & \\
\hline & High & 47 & $1.993(1.045-3.799)$ & $0.036^{*}$ & $3.479(1.553-7.795)$ & $0.002^{* *}$ \\
\hline
\end{tabular}

Abbreviations: $\mathrm{HR}=$ hazard ratio; $95 \% \mathrm{CI}=95 \%$ confidence interval. ${ }^{\mathrm{T} X X}$ and $\mathrm{NX}$ : samples with unknown pathological tumor or node stage. ${ }^{*} P<0.05,{ }^{* *} P<0.01$.

A

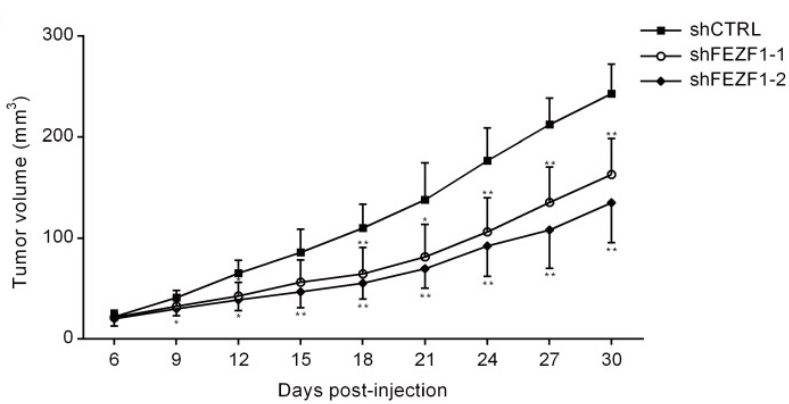

B
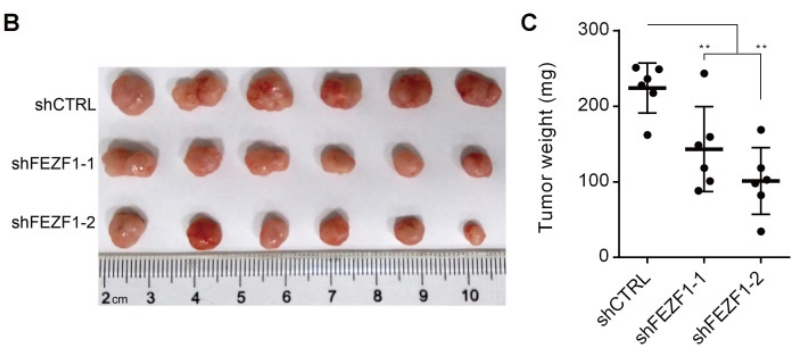

Figure 3. FEZF1 knockdown inhibited C33A cell growth in vivo. Tumorigenesis assay was performed by subcutaneous injection of FEZF1 knockdown and control C $33 \mathrm{~A}$ cells $\left(5 \times 10^{6}\right)$ into right flanks of $\mathrm{BALB} / \mathrm{c}$ nude mice. (A) Tumor volumes were measured at the indicated days post-injection. (B, C) Tumors were photographed and weighted at 30 days. Data represent mean $\pm \mathrm{SD}, \mathrm{n}=6 . * P<0.05, * * P<0.01$

\section{FEZFI knockdown inhibited cell growth in vivo}

To test FEZF1 function in vivo, we performed tumorigenesis assay by subcutaneous injection of C33A cells into right flanks of nude mice. In the course of tumor development, the growth of tumors was decelerated by FEZF1 knockdown in C33A cells (Figure $3 \mathrm{~A})$. At 30 days post-injection, tumors were dissected and weighted. Tumors generated by FEZF1 knockdown C33A cells were much smaller and lighter than the tumors of control cells (Figure $3 \mathrm{~B}$ and 3C). The results indicated that FEZF1 has the ability to promote cervical cancer cell growth in vivo.

\section{Expression of FEZFI promoted cell proliferation, growth and migration in HeLa cells}

To further confirm the function of FEZF1, we ectopically expressed a FLAG tagged EGFP-FEZF1 fusion protein in HeLa cells (Figure 4A), in which FEZF1 is not expressed endogenously. The effect of FEZF1 on cell proliferation was examined by CCK-8 assay. The result showed that the expression of FEZF1 promoted cell proliferation in HeLa cells (Figure 4B). Meanwhile, FEZF1-expressing HeLa cells formed 
more colonies than the control cells in colony formation assay (Figure 4C). In addition, Transwell assay revealed that cell migration ability was also enhanced by FEZF1 expression in HeLa cells (Figure 4D).

\section{FEZF 1 bound to multiple key genes in the Wnt signaling pathway in HeLa cells}

In a recent study that characterized $\mathrm{C} 2 \mathrm{H} 2$ zinc finger proteins, the genomic binding sites of FEZF1 were determined in HEK293 cells by ChIP-seq analysis [16]. To gain insight into the molecular function of FEZF1, we preformed GO enrichment analysis for FEZF1 target genes. In consistence with its function in nervous system development, the result showed that FEZF1 mainly binds to genes involved in neuronal differentiation, epithelial cell migration and developmental growth, as well as genes in the Wnt signaling pathway (Figure 5A). The binding of FEZF1 to the Wnt signaling pathway genes was visualized by the Pathview package based on KEGG pathways. As shown in Figure 5B, FEZF1 bound to dozens of genes in both canonical and non-canonical Wnt pathways. To determine whether FEZF1 binds to these Wnt pathway genes in cervical cancer cells, we performed ChIP assay in HeLa cells expressing the FLAG tagged EGFP-FEZF1 protein. Consistent with the ChIP-seq study in HEK293 cells, the result showed that FEZF1 bound to multiple key Wnt pathway genes in HeLa cells (Figure 5C), suggesting a regulatory role of FEZF1 in the Wnt pathway in cervical cancer cells.

\section{FEZF 1 activated the Wnt signaling pathway in cervical cancer cells}

We further investigated the effects of FEZF1 on the Wnt signaling pathway in cervical cancer cells. $\beta$-catenin protein, encoded by CTNNB1 gene, is the core component of the canonical Wnt pathway (also known as Wnt/ $\beta$-catenin pathway). Upon activation by upstream signals, $\beta$-catenin accumulates, binds to and activates TCF/LEF (T-cell factor/lymphoid enhancing factor) transcription factors in the nucleus, which leads to the activation of the downstream genes that are involved in cell proliferation and growth, such as JUN and CCND1. Therefore, we first measured the effect of FEZF1 on the mRNA and protein levels of $\beta$-catenin by RT-qPCR and western blot. The results showed that, although the mRNA level was not changed (Figure 6A and 6B, left), the level of $\beta$-catenin protein was reduced by FEZF1 knockdown in C33A cells, whereas increased by FEZF1 expression in HeLa cells (Figure 6A and 6B, right). In addition, we also measured the expression of TCF7L2 gene which encodes TCF4, a common protein of the $\beta$-catenin-TCF complex, and two downstream effector genes, JUN and CCND1. As expected, the levels of these genes were decreased upon knockdown of FEZF1 in C33A cells, while increased by the expression of FEZF1 in HeLa cells (Figure 6C and 6D).

The two non-canonical pathways, the Wnt/ planar cell polarity $(\mathrm{PCP})$ pathway and the $\mathrm{Wnt} / \mathrm{Ca}^{2+}$ pathway, does not involve $\beta$-catenin. We selected four downstream effector genes in these two pathways and determined the effect of FEZF1 on their expression. The results showed that these genes were also downregulated in FEZF1 knockdown C33A cells, while upregulated in FEZF1-expressing HeLa cells (Figure $6 \mathrm{E}$ and $6 \mathrm{~F}$ ). Together, these results suggested that FEZF1 is a positive regulator of both canonical and non-canonical Wnt signaling pathways in cervical cancer cells.

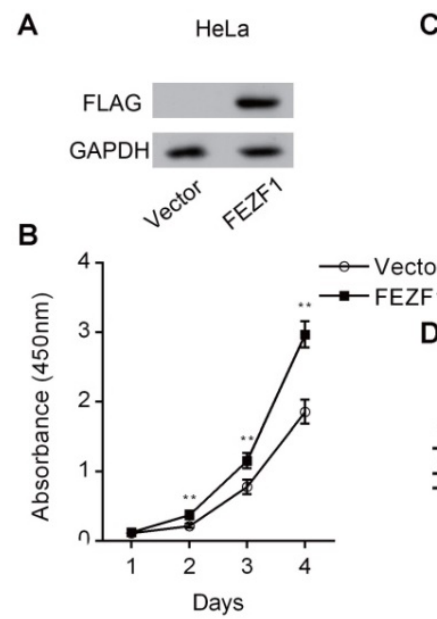

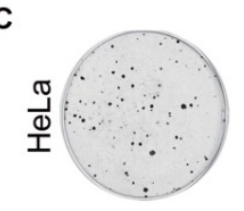

Vector

D

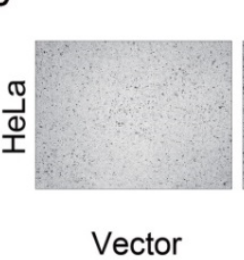

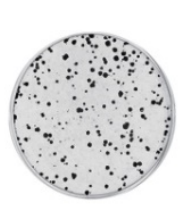

FEZF1
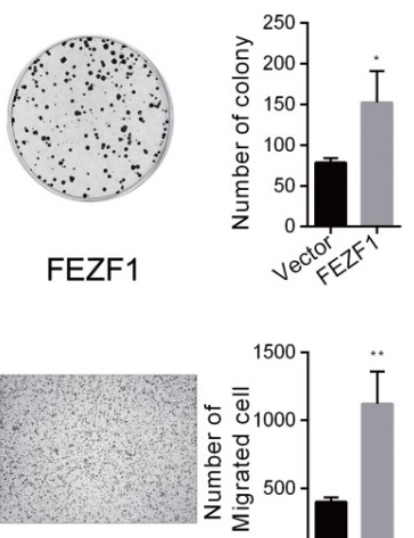

FEZF1

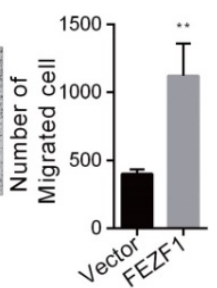

Figure 4. Expression of FEZFI promoted cell proliferation, growth and migration in HeLa cells. (A) Western blot assay showed the ectopically expression of a FLAG tagged EGFP-FEZF1 fusion protein in HeLa cells. (B) Cell Counting Kit-8 assay demonstrated that cell proliferation was increased significantly in HeLa cells expressing FEZF1 protein. (C) Colony formation assay showed that FEZF1-expressing HeLa cells formed more colonies compared to the control cells. (D) Transwell migration assay revealed that expression of FEZF1 promoted cell migration in HeLa cells. Data represent mean \pm SD of three independent experiments. $* P<0.05$, $* * P<0.01$. 
A

cell morphogenesis involved in neuron differentiation-

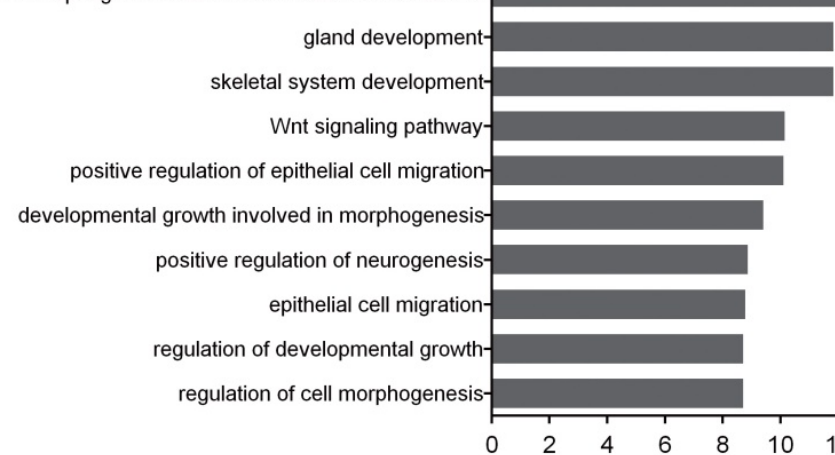

B
C

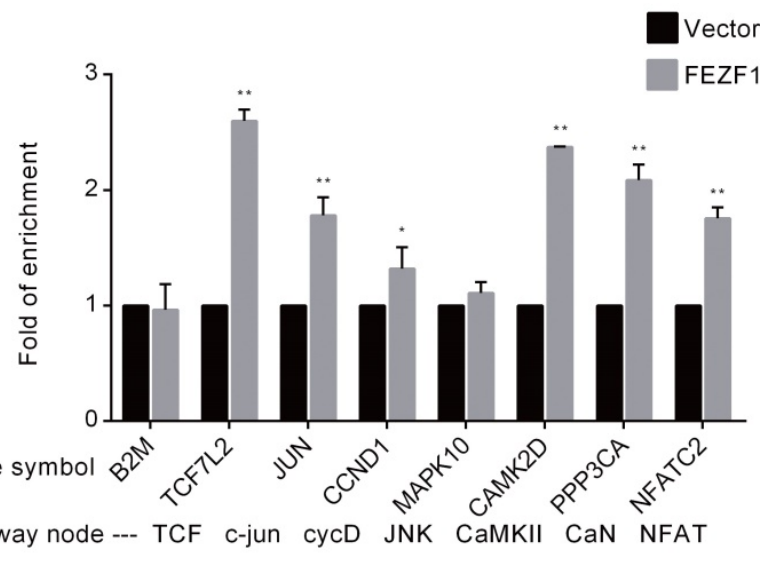

p.adjust $\left(-\log _{10}\right)$

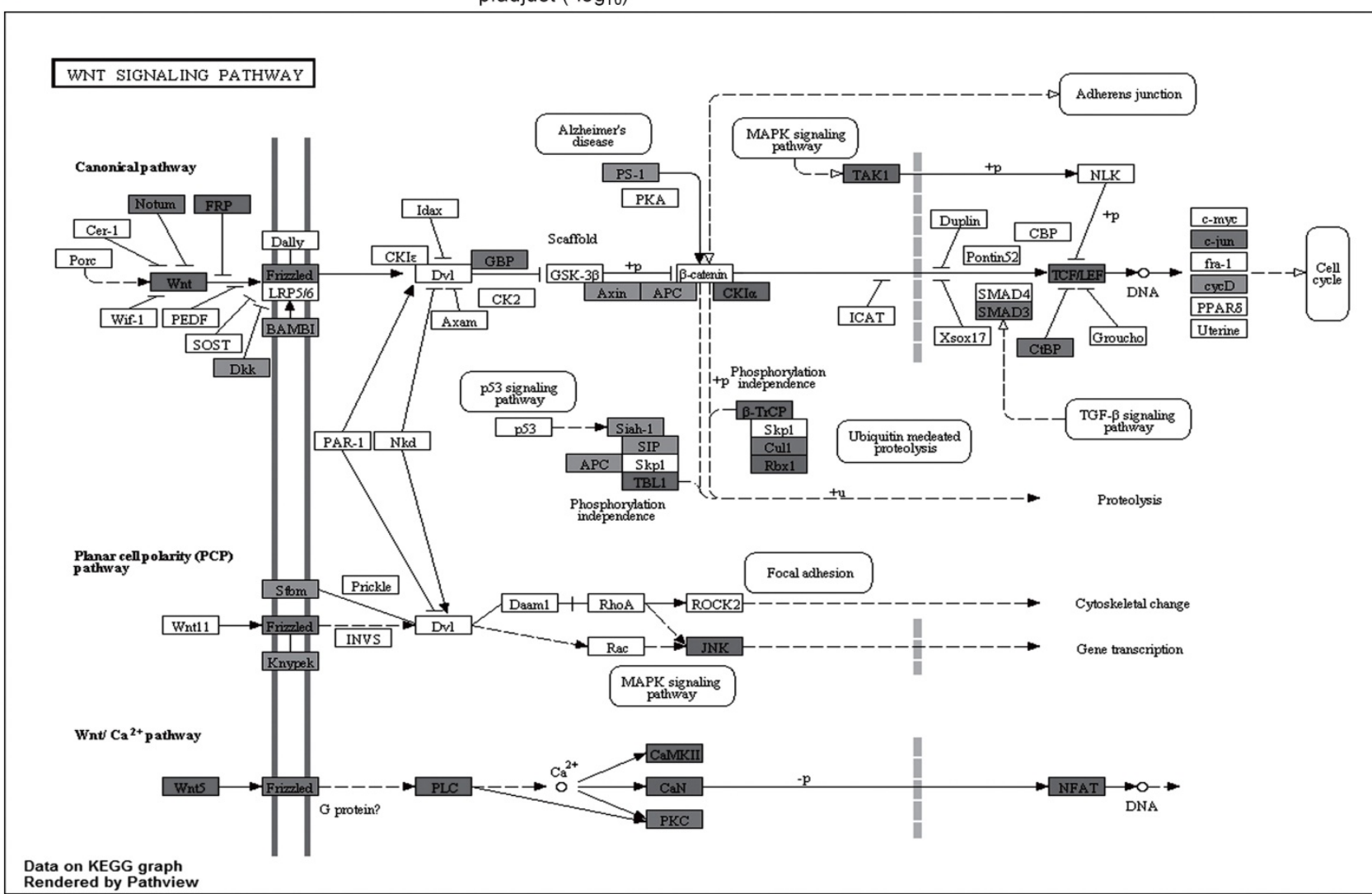

Figure 5. FEZFI bound to genes in the Wnt signaling pathway. (A) GO enrichment analysis of the FEZF1-binding genes determined by a previous ChIP-seq assay in HEK293 cells. (B) Visualization of the previously determined FEZF1-binding genes in the Wnt signaling pathway using the Pathview package based on KEGG pathways. Dark gray indicates strong binding of FEZFI to target genes. (C) ChIP assay demonstrated the binding of FEZF1 to the Wnt pathway genes in HeLa cells. B2M promoter region was used as a control for non-specific binding. Data represent mean \pm SD of three independent experiments. $* P<0.05, * * P<0.01$.

\section{Discussion}

Recurrence is a difficult clinical problem for cervical cancer, as most of patients with relapse are not curable [6, 7]. Optimization of primary treatment, based on the risk of recurrence, could be more rewarding than a deliberate post-treatment surveillance or aggressive salvage therapy [8]. Currently, clinicopathological factors, including tumor diameter, depth of stromal invasion, lymphvascular space invasion, parametrial extension, histologic variants, lymph node involvement and status of the resection margins, are used individually or in combination to evaluate the risk of recurrence in early-stage patients [17]. Patients who are deemed to be at high risk should receive adjuvant radiation therapy with or without chemotherapy after surgery [3]. However, the prognostic value of these clinicopathological factors is controversial, as these factors are lacking in sensitivity and reproducibility, which is mainly attributed to the wide variations in clinicopathological definitions and criteria.[17]. The squamous cell carcinoma antigen (SCC-Ag), a common serum protein marker, has also 
been assessed for recurrence prediction in cervical cancer [18]. Elevated post-treatment SCC-Ag level can precede clinical diagnosis in $46-92 \%$ of the cases, with a mean lead time ranging from 2 to 8 months, but the prognostic value of pre-treatment serum SCC-Ag on recurrence prediction is still debated [18]. In this study, we found that the level of FEZF1 in the primary tumor samples of cervical cancer was an independent diagnostic factor for recurrence using TCGA datasets. Patients with high level of FEZF1 were at significantly higher risk of recurrence $(\mathrm{HR}=3.479 ; 95 \% \mathrm{CI}$, 1.553-7.795; $P=0.002$ ) than patients with low level of FEZF1 expression (Table 2). The introduction of FEZF1 as a predictive molecular marker for recurrence may facilitate the optimization of primary treatment and improve the overall outcomes of cervical cancer patients.

In addition, a significant increase of FEZF1 expression was observed in the primary tumor samples from patients with relapse (Figure 1C). In addition, expression of FEZF1 in HeLa cells enhanced cell proliferation, growth and migration (Figure 4), while knockdown of FEZF1 has the opposite effects on both C33A and SiHa cells (Figure 2). Meanwhile, FEZF1 knockdown also inhibited tumor growth in vivo (Figure 3). All these results demonstrated that FEZF1 plays an oncogenic role in cervical cancer.

The Wnt signaling pathway controls body axis patterning, cell fate specification, cell proliferation and cell migration during development, and aberrant
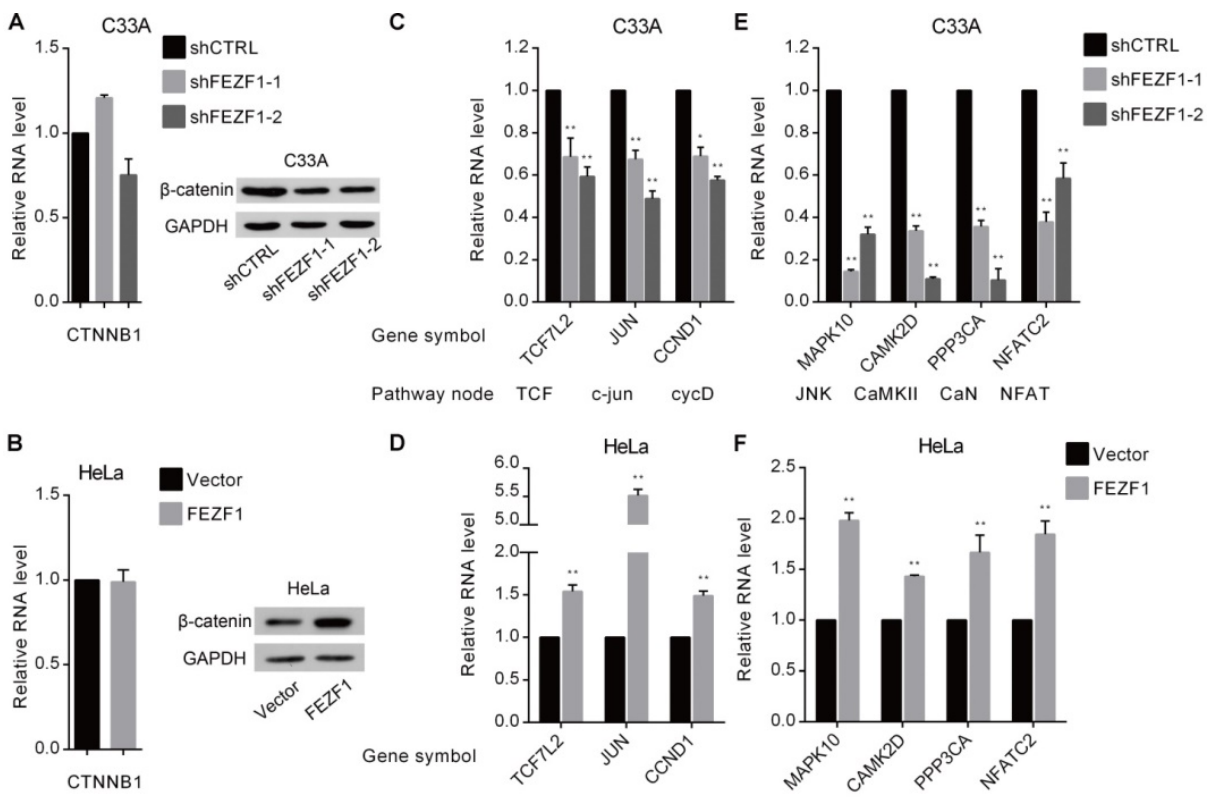

D
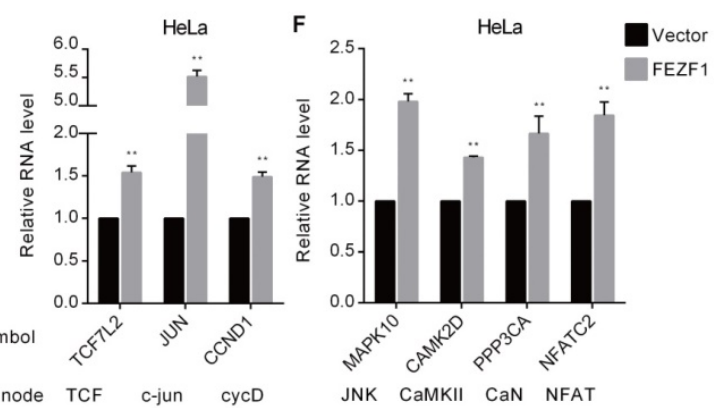

Figure 6. FEZF1 activated the Wnt signaling pathway in cervical cancer cells. (A, B) RT-qPCR and western blot analysis of $\beta$-catenin mRNA (left) and protein (right) levels in FEZFI knockdown C33A and FEZF1-expressing HeLa cells. (C, D) RT-qPCR analysis of the expression of $\beta$-catenin downstream genes in FEZF1 knockdown C33A and FEZFI-expressing HeLa cells. The Wnt pathway nodes of corresponding genes are indicated below. (E, F) RT-qPCR analysis of selected non-canonical Wnt pathway genes in FEZFI knockdown C33A cells and FEZFI-expressing HeLa cells. Data represent mean \pm SD of three independent experiments. $* P<0.05, * * P<0.01$. activation of this pathway has been implicated in the development of many types of human cancers [19-21], including cervical cancer [22]. Increase of $\beta$-catenin, the hallmark of canonical Wnt pathway activation, has been reported to be an independent prognostic factor for the RFS and OS of cervical cancer patients [23]. Although FEZF1 does not bind to CTNNB1 gene directly, many upstream pathway genes and several genes that control $\beta$-catenin stability are FEZF1 targets (Figure 5B). In this study, we found that the level of $\beta$-catenin protein was increased by the expression of FEZF1 in HeLa cells, while reduced by FEZF1 knockdown in C33A cells (Figure 6A and 6B). The results indicated that FEZF1 could activate the canonical Wnt pathway in cervical cancer cells. Moreover, the binding of FEZF1 to multiple key Wnt genes was also confirmed by ChIP assay (Figure 5C), and the effects of FEZF1 on their expression were examined by RT-qPCR (Figure 6C-F). These results showed that FEZF1 could bind to and activate TCF7L2 gene which encodes TCF4, a common effector of $\beta$-catenin. Meanwhile, the upregulation of two important downstream effector genes, JUN and CCND1, further confirmed the positive regulatory role of FEZF1 in the canonical Wnt signaling pathways. Although not as well characterized as the canonical pathways, the two non-canonical pathways have also been found to be involved in cancer progression [24, 25]. In this study, we found that FEZF1 also bound to and activated key genes, such as CaMK2D, PPP3CA and NFATC2, in the non-canonical Wnt signaling pathways. Therefore, we propose that FEZF1 is a transcriptional activator for the Wnt signaling pathways in cervical cancer.

In summary, our results suggest that FEZF1 plays an oncogenic role in cervical cancer possibly by acting as a transcription activator of the Wnt signaling pathway. Since the Wnt signaling pathway has been considered as a promising therapeutic target in cancer [26], we suppose that FEZF1 may serve a potential target for preventing recurrence in cervical cancer. Importantly, we also introduced FEZF1 as a valuable predictive marker 
for cervical cancer recurrence, which may greatly improve the management of cervical cancer.

\section{Supplementary Material}

Supplementary figures and tables. http://www.jcancer.org/v09p3929s1.pdf

\section{Acknowledgements}

This work was supported by National Key R\&D Program of China (2017YFA0504300), National Natural Science Foundation of China (31771441, 81490752, 31371325, 31671347, 31000579, and 30971634), Doctoral Programs Foundation of the Ministry of Education, China (20130181130010).

\section{Competing Interests}

The authors have declared that no competing interest exists.

\section{References}

1. Torre LA, Bray F, Siegel RL, et al. Global cancer statistics, 2012. Ca-Cancer J Clin. 2015; 65: 87-108.

2. Canavan TP, Doshi NR. Cervical cancer. Am Fam Physician. 2000; 61: 1369-1376.

3. Petignat P, Roy M. Diagnosis and management of cervical cancer. BMJ. 2007; 335: 765-768.

4. Duyn A, Van Eijkeren M, Kenter G, et al. Recurrent cervical cancer: detection and prognosis. Acta Obstet Gynecol Scand. 2002; 81: 759-763.

5. Elit L, Fyles AW, Devries MC, et al. Follow-up for women after treatment for cervical cancer: a systematic review. Gynecol Oncol. 2009; 114: 528-535.

6. Wang CJ, Lai CH, Huang HJ, et al. Recurrent cervical carcinoma after primary radical surgery. Am J Obstet Gynecol. 1999; 181: 518-524.

7. Qiu JT, Abdullah NA, Chou HH, et al. Outcomes and prognosis of patients with recurrent cervical cancer after radical hysterectomy. Gynecol Oncol. 2012; 127: $472-477$.

8. Dreyer G, Snyman LC, Mouton A, et al. Management of recurrent cervical cancer. Best Pract Res Clin Obstet Gynaecol. 2005; 19: 631-644.

9. Eckler MJ, Chen B. Fez family transcription factors: controlling neurogenesis and cell fate in the developing mammalian nervous system. BioEssays. 2014; 36: 788-797.

10. Shimizu T, Nakazawa M, Kani S, et al. Zinc finger genes Fezf1 and Fezf2 control neuronal differentiation by repressing Hes 5 expression in the forebrain. Development. 2010; 137: 1875-1885.

11. Song IS, Oh NS, Kim HT, et al. Human ZNF312b promotes the progression of gastric cancer by transcriptional activation of the K-ras gene. Cancer Res. 2009; 69: 3131-3139.

12. Chen $\mathrm{N}$, Guo $\mathrm{D}, \mathrm{Xu} \mathrm{Q}$, et al. Long non-coding RNA FEZF1-AS1 facilitates cell proliferation and migration in colorectal carcinoma. Oncotarget. 2016; 7: 11271-11283.

13. Yu G, Wang LG, Han Y, et al. clusterProfiler: an R package for comparing biological themes among gene clusters. OMICS. 2012; 16: 284-287.

14. Yu G, Li F, Qin $\mathrm{Y}$, et al. GOSemSim: an R package for measuring semantic similarity among GO terms and gene products. Bioinformatics. 2010; 26: 976-978.

15. Luo W, Brouwer C. Pathview: an R/Bioconductor package for pathway-based data integration and visualization. Bioinformatics. 2013; 29: 1830-1831.

16. Schmitges FW, Radovani E, Najafabadi HS, et al. Multiparameter functional diversity of human $\mathrm{C} 2 \mathrm{H} 2$ zinc finger proteins. Genome Res. 2016; 26: $1742-1752$.

17. Singh N, Arif S. Histopathologic parameters of prognosis in cervical cancer--a review. Int J Gynecol Cancer. 2004; 14: 741-750.

18. Gadducci A, Tana R, Cosio S, et al. The serum assay of tumour markers in the prognostic evaluation, treatment monitoring and follow-up of patients with cervical cancer: a review of the literature. Crit Rev Oncol Hematol. 2008; 66: $10-20$.

19. Reya T, Clevers H. Wnt signalling in stem cells and cancer. Nature. 2005; 434: 843-850.

20. Polakis P. Wnt signaling in cancer. Cold Spring Harbor Perspect Biol. 2012; 4: a008052.

21. Klaus A, Birchmeier W. Wnt signalling and its impact on development and cancer. Nat Rev Cancer. 2008; 8: 387-398.

22. Bahrami A, Hasanzadeh M, ShahidSales S, et al. Clinical Significance and Prognosis Value of Wnt Signaling Pathway in Cervical Cancer. J Cell Biochem. 2017; 118: 3028-3033.
23. Liang J, Zhou H, Peng Y, et al. $\beta$-Catenin Expression Negatively Correlates with WIF1 and Predicts Poor Clinical Outcomes in Patients with Cervical Cancer. BioMed Res Int. 2016; 2016: 4923903.

24. Wang Y. Wnt/Planar cell polarity signaling: a new paradigm for cancer therapy. Mol Cancer Ther. 2009; 8: 2103-2109.

25. De A. Wnt/Ca2+ signaling pathway: a brief overview. Acta Biochim Biophys Sin. 2011; 43: 745-756.

26. Anastas JN, Moon RT. WNT signalling pathways as therapeutic targets in cancer. Nat Rev Cancer. 2013; 13: 11-26. 